\title{
A bound of sums with convolutions of Dirichlet characters
}

\author{
Teerapat Srichan \\ Department of Mathematics, Faculty of Science \\ Kasetsart University, Bangkok, Thailand \\ e-mail: fscitrp@ku.ac.th
}

Received: 16 July 2019

Revised: 10 February 2020

Accepted: 24 February 2020

Abstract: We use the exponent pair to bound sums $\sum_{a b \leq x} \chi_{1}(a) \chi_{2}(b)$, where $\chi_{1}$ and $\chi_{2}$ are primitive Dirichlet characters with conductors $q_{1}$ and $q_{2}$, respectively.

Keywords: Character sums, Dirichlet convolutions, Exponent pair.

2010 Mathematics Subject Classification: Primary: 11L07; Secondary: 11N37, 11M06.

\section{Introduction and statement of result}

Let $\chi_{1}$ and $\chi_{2}$ be two primitive Dirichlet characters with conductors $q_{1}$ and $q_{2}$, respectively. For $x>1$, define

$$
S_{\chi_{1}, \chi_{2}}(x)=\sum_{a b \leq x} \chi_{1}(a) \chi_{2}(b)
$$

The sum (1) is a generalized form of the character sum. A symptotic formulas for (1) can found in [2] and [3]. In 2010 Banks and Shparlinski [1] bounded $S_{\chi_{1}, \chi_{2}}(x)$ for small values of $x$ and proved that, if $x \geq q_{2}^{2 / 3} \geq q_{1}^{2 / 3}$ and $\log x=q_{2}^{o(1)}$, then

$$
\left|S_{\chi_{1}, \chi_{2}}(x)\right| \leq x^{13 / 18} q_{1}^{2 / 27} q_{2}^{1 / 9+o(1)},
$$

and if $x \geq q_{2}^{3 / 4} \geq q_{1}^{3 / 4}$ and $\log x=q_{2}^{o(1)}$, then

$$
\left|S_{\chi_{1}, \chi_{2}}(x)\right| \leq x^{5 / 18} q_{1}^{3 / 32} q_{2}^{3 / 16+o(1)} .
$$


Banks and Shparlinski combined the Polya-Vinogranov bound with the Burgess bounds to prove (2) and (3).

In this paper we shall provide another bound for the sum in (1) by using exponent pairs. Our result is following;

Theorem 1.1. Let $\chi_{1}$ and $\chi_{2}$ be two primitive Dirichlet characters with conductors $q_{1}$ and $q_{2}$, respectively. For $x \geq q_{i}^{2}, i=1,2$, we have

$$
S_{\chi_{1}, \chi_{2}}(x)=O\left(x^{1 / 3} q_{1}^{5 / 9} q_{2}^{7 / 9} \log q_{1}\right) .
$$

\section{Prerequisites}

Notation. Throughout this paper $\epsilon$ denotes a fixed positive constant, not necessarily the same in all occurrenes. Let $\psi(x)=x-\lfloor x\rfloor-\frac{1}{2}$. For $r=1,2, \ldots$ the exponent pair is

$$
\left(k_{r}, l_{r}\right)=\left(\frac{1}{2}-\frac{r+1}{2(2 \Lambda-1)}, \frac{1}{2}+\frac{1}{2(2 \Lambda-1)}\right), \Lambda=2^{r} .
$$

The following lemmas are needed in our proof.

Lemma 2.1. Let $\chi$ be a primitive character modulo $q$. For a real $z>1$, we have

$$
\sum_{a \leq z} \chi(a)=\sum_{j \leq q} \chi(j)\left\lfloor\frac{z}{q}-\frac{j}{q}+1\right\rfloor
$$

Proof. From the periodicity of the primitive character modulo $q$, we have

$$
\sum_{a \leq z} \chi(a)=\sum_{j \leq q} \sum_{\substack{a \leq z \\ a \equiv j(\bmod q)}} \chi(a)=\sum_{j \leq q} \sum_{\substack{a \leq z \\ a \equiv j(\bmod q)}} \chi(j)=\sum_{j \leq q} \chi(j) \sum_{\substack{a \leq z \\ a \equiv j(\bmod q)}} 1=\sum_{j \leq q} \chi(j)\left\lfloor\frac{z}{q}-\frac{j}{q}+1\right\rfloor .
$$

Lemma 2.2 (see [4, Lemma 17]). Let $x, \eta, \alpha, \omega$ be real numbers, $j$ and $q$ be positive numbers, where $x \geq 1, \alpha>0, \eta \geq 1,1 \leq j \leq q$, and $(k, l)$ is an exponent pair with $k>0$ and

$$
R(x, \eta, \alpha ; q, j ; \omega)=\sum_{\substack{n \leq \eta \\ n \equiv j(\bmod q)}} \psi\left(\frac{x}{n^{\alpha}}+\omega\right)
$$

if $\omega$ is independent on $n$. Then

$$
R(x, \eta, \alpha ; q, j ; \omega)=O(1)+O\left(x^{\frac{-1}{2}} \eta^{1+\frac{\alpha}{2}} q^{-1}\right)+ \begin{cases}O\left(x^{\frac{k}{k+1}} \eta^{\frac{l-\alpha k}{k+1}} q^{\frac{-l}{k+1}}\right) & \text { for } l>\alpha k \\ O\left(x^{\frac{k}{k+1}} \log \eta q^{\frac{-\alpha k}{k+1}}\right) & \text { for } l=\alpha k \\ O\left(\left(x q^{-\alpha}\right)^{\frac{k}{1+(1+\alpha) k-l}}\right) & \text { for } l<\alpha k,\end{cases}
$$

where the O-Constants is dependent on only $\alpha$. 


\section{Proof of Theorem 1.1}

Proof. For $x>1$, we have

$$
S_{\chi_{1}, \chi_{2}}(x)=\sum_{a \leq x^{1 / 2}} \chi_{1}(a) \sum_{b \leq x / a} \chi_{2}(b)+\sum_{b \leq x^{1 / 2}} \chi_{2}(b) \sum_{a \leq x / b} \chi_{1}(a)-\sum_{a \leq x^{1 / 2}} \chi_{1}(a) \sum_{b \leq x^{1 / 2}} \chi_{2}(b) .
$$

In view of Lemma 2.1 we have

$$
S_{\chi_{1}, \chi_{2}}(x)=E_{1}+E_{2}-E_{3},
$$

where

$$
\begin{aligned}
& E_{1}=\sum_{j \leq q_{2}} \chi_{2}(j) \sum_{a \leq x^{1 / 2}} \chi_{1}(a)\left\lfloor\frac{x}{a q_{2}}-\frac{j}{q_{2}}+1\right\rfloor, \\
& E_{2}=\sum_{h \leq q_{1}} \chi_{1}(h) \sum_{b \leq x^{1 / 2}} \chi_{2}(b)\left\lfloor\frac{x}{b q_{1}}-\frac{h}{q_{1}}+1\right\rfloor, \\
& E_{3}=\sum_{h \leq q_{1}} \chi_{1}(h) \sum_{j \leq q_{2}} \chi_{2}(j)\left\lfloor\frac{x^{1 / 2}}{q_{1}}-\frac{h}{q_{1}}+1\right\rfloor\left\lfloor\frac{x^{1 / 2}}{b q_{2}}-\frac{j}{q_{2}}+1\right\rfloor .
\end{aligned}
$$

From $\lfloor x\rfloor=x-\psi(x)-\frac{1}{2}, \psi(x)=\psi(x+1)$ and the identity $\sum_{j \leq q_{i}} \chi_{i}(j)=0$, for $i=1$, we have

$$
\begin{aligned}
E_{1} & =\sum_{j \leq q_{2}} \chi_{2}(j) \sum_{a \leq x^{1 / 2}} \chi_{1}(a)\left(\frac{x}{a q_{2}}-\frac{j}{q_{2}}+\frac{1}{2}-\psi\left(\frac{x}{a q_{2}}-\frac{j}{q_{2}}\right)\right) \\
& =-\sum_{j \leq q_{2}} \chi_{2}(j) \sum_{a \leq x^{1 / 2}} \chi_{1}(a)\left(\frac{j}{q_{2}}+\psi\left(\frac{x}{a q_{2}}-\frac{j}{q_{2}}\right)\right) \\
& =-\frac{1}{q_{2}} \sum_{j \leq q_{2}} j \chi_{2}(j) \sum_{a \leq x^{1 / 2}} \chi_{1}(a)-\sum_{j \leq q_{2}} \chi_{2}(j) \sum_{a \leq x^{1 / 2}} \chi_{1}(a) \psi\left(\frac{x}{a q_{2}}-\frac{j}{q_{2}}\right) .
\end{aligned}
$$

In view of Lemma 2.1 and the periodicity of the character modulo $q$, we have

$$
\begin{aligned}
E_{1}= & -\frac{1}{q_{2}} \sum_{\substack{h \leq q_{1} \\
j \leq q_{2}}} j \chi_{1}(h) \chi_{2}(j)\left\lfloor\frac{x^{1 / 2}}{q_{1}}-\frac{h}{q_{1}}+1\right\rfloor \\
& -\sum_{\substack{h \leq q_{1} \\
j \leq q_{2}}} \chi_{1}(h) \chi_{2}(j) \sum_{\substack{a \leq x^{1 / 2} \\
a \equiv h\left(\bmod q_{1}\right)}} \psi\left(\frac{x}{a q_{2}}-\frac{j}{q_{2}}\right) .
\end{aligned}
$$

For $E_{2}$ and $E_{3}$, computation similar to $E_{1}$ yields,

$$
\begin{aligned}
E_{2}= & -\frac{1}{q_{1}} \sum_{\substack{h \leq q_{1} \\
j \leq q_{2}}} h \chi_{1}(h) \chi_{2}(j)\left\lfloor\frac{x^{1 / 2}}{q_{2}}-\frac{j}{q_{2}}+1\right\rfloor \\
& -\sum_{\substack{h \leq q_{1} \\
j \leq q_{2}}} \chi_{1}(h) \chi_{2}(j) \sum_{\substack{a \leq x^{1 / 2} \\
a \equiv j\left(\bmod q_{2}\right)}} \psi\left(\frac{x}{a q_{1}}-\frac{h}{q_{1}}\right),
\end{aligned}
$$


and

$$
\begin{aligned}
E_{3}= & -\frac{1}{q_{2}} \sum_{\substack{h \leq q_{1} \\
j \leq q_{2}}} j \chi_{1}(h) \chi_{2}(j)\left\lfloor\frac{x^{1 / 2}}{q_{1}}-\frac{h}{q_{1}}+1\right\rfloor \\
& -\frac{1}{q_{1}} \sum_{\substack{h \leq q_{1} \\
j \leq q_{2}}} h \chi_{1}(h) \chi_{2}(j)\left\lfloor\frac{x^{1 / 2}}{q_{2}}-\frac{j}{q_{2}}+1\right\rfloor+O\left(q_{1} q_{2}\right) .
\end{aligned}
$$

Thus, we have

$$
\begin{aligned}
S_{\chi_{1}, \chi_{2}}(x)= & -\sum_{\substack{h \leq q_{1} \\
j \leq q_{2}}} \chi_{1}(h) \chi_{2}(j) \sum_{\substack{a \leq x^{1 / 2} \\
a \equiv h\left(\bmod q_{1}\right)}} \psi\left(\frac{x}{a q_{2}}-\frac{j}{q_{2}}\right) \\
& -\sum_{\substack{h \leq q_{1} \\
j \leq q_{2}}} \chi_{1}(h) \chi_{2}(j) \sum_{\substack{a \leq x^{1 / 2} \\
a \equiv j\left(\bmod q_{2}\right)}} \psi\left(\frac{x}{a q_{1}}-\frac{h}{q_{1}}\right)+O\left(q_{1} q_{2}\right) .
\end{aligned}
$$

In view of Lemma 2.2, for the exponent pair $(2 / 7,4 / 7)$, we have

$$
\begin{aligned}
& \sum_{\substack{h \leq q_{1} \\
j \leq q_{2}}} \chi_{1}(h) \chi_{2}(j) \sum_{\substack{a \leq x^{1 / 2} \\
a \equiv h=\left(\bmod q_{1}\right)}} \psi\left(\frac{x}{a q_{2}}-\frac{j}{q_{2}}\right) \\
& =\sum_{\substack{h \leq q_{1} \\
j \leq q_{2}}} \chi_{1}(h) \chi_{2}(j) R\left(\frac{x}{q_{2}}, x^{1 / 2}, 1, q_{1}, h, \frac{-j}{q_{2}}\right) \\
& \ll \sum_{\substack{h \leq q_{1} \\
j \leq q_{2}}}\left(1+\frac{x^{1 / 4} q_{2}^{1 / 2}}{q_{1}}+\frac{x^{1 / 3}}{q_{2}^{2 / 9} q_{1}^{4 / 9}}\right) \\
& =O\left(q_{1} q_{2}+x^{1 / 4} q_{2}^{3 / 2} \log q_{1}+x^{1 / 3} q_{1}^{5 / 9} q_{2}^{7 / 9}\right) .
\end{aligned}
$$

In the same way, we have

$$
\sum_{\substack{h \leq q_{1} \\ j \leq q_{2}}} \chi_{1}(h) \chi_{2}(j) \sum_{\substack{a \leq x^{1 / 2} \\ a \equiv j\left(\bmod q_{2}\right)}} \psi\left(\frac{x}{a q_{1}}-\frac{h}{q_{1}}\right)=O\left(q_{1} q_{2}+x^{1 / 4} q_{1}^{3 / 2} \log q_{2}+x^{1 / 3} q_{2}^{5 / 9} q_{1}^{7 / 9}\right) .
$$

Thus, we have

$$
S_{\chi_{1}, \chi_{2}}(x)=O\left(x^{1 / 4} q_{2}^{3 / 2} \log q_{1}+x^{1 / 3} q_{1}^{5 / 9} q_{2}^{7 / 9}\right)+O\left(x^{1 / 4} q_{1}^{3 / 2} \log q_{2}+x^{1 / 3} q_{2}^{5 / 9} q_{1}^{7 / 9}\right)+O\left(q_{1} q_{2}\right) .
$$

We note that, for $x \geq q_{1}^{2}, q_{2}^{2}$, the error term $x^{1 / 3} q_{1}^{5 / 9} q_{2}^{7 / 9} \log q_{1}$ dominates the remaining terms. Thus, for $x \geq q_{1}^{2}, q_{2}^{2}$, we obtain the result in Theorem 1.1 .

\section{Acknowledgements}

We thank the referees for their time and comments. This work was supported by Department of Mathematics, Faculty of Science, Kasetsart University, Bangkok Thailand. 


\section{References}

[1] Banks, W. D., \& Shparlinski, I. E. (2010). Sums with convolutions of Dirichlet characters, Manuscripta Math. 133, 105-144.

[2] Friedlander, J. B., \& Iwaniec, H. (2005). Summation formulae for coefficients of $L$-functions, Canad. J. Math. 57, 494-505.

[3] Iwaniec, H., \& Kowalski, E. (2004). Analytic Number Theory, American Mathematical Society, Providence.

[4] Richert, H. E. (1953). Über die Anzahl Abelscher Gruppen gegebener Ordnung. II., Math. Z., 58 (1), 71-84. 